Irish Math. Soc. Bulletin

Number 86, Winter 2020, 103-106

ISSN 0791-5578

\title{
Goldbach's Conjecture: if it's unprovable, it must be true
}

\author{
PETER LYNCH
}

\begin{abstract}
Goldbach's Conjecture is one of the best-known unsolved problems in mathematics. Over the past 280 years, many brilliant mathematicians have tried and failed to prove it. If a proof is found, it will likely involve some radically new idea or approach. If the conjecture is unprovable using the usual axioms of set theory, then it must be true. This is because, if a counter-example exists, it can be found by a finite search.
\end{abstract}

In 1742, Christian Goldbach wrote a letter to Leonhard Euler proposing that every integer greater than 2 is the sum of three primes. Euler responded that this would follow from the simpler statement that every even integer greater than 2 is the sum of two primes.

Goldbach's Conjecture is one of the best-known unsolved problems in mathematics. It is a simple matter to check the conjecture for the first few cases:

$$
\begin{array}{rcc}
4=2+2 & 6=3+3 & 8=5+3 \\
10=7+3 & 12=7+5 & 14=11+3 \\
16=13+3 & 18=13+5 & 20=17+3 \\
\ldots & \ldots & \ldots
\end{array}
$$

But, with an infinite number of cases, this approach can never prove the conjecture.

If, for an even number $2 n$ we have $2 n=p_{1}+p_{2}$ where $p_{1}$ and $p_{2}$ are primes, then obviously

$$
n=\frac{p_{1}+p_{2}}{2}
$$

If every even number can be partitioned in this way, then any number, even or odd, must be the arithmetic average, or mean, of two primes.

In Fig. 1 we plot the natural numbers from 1 to 25 (blue) and the first nine primes (red) in rows above and below the number line.

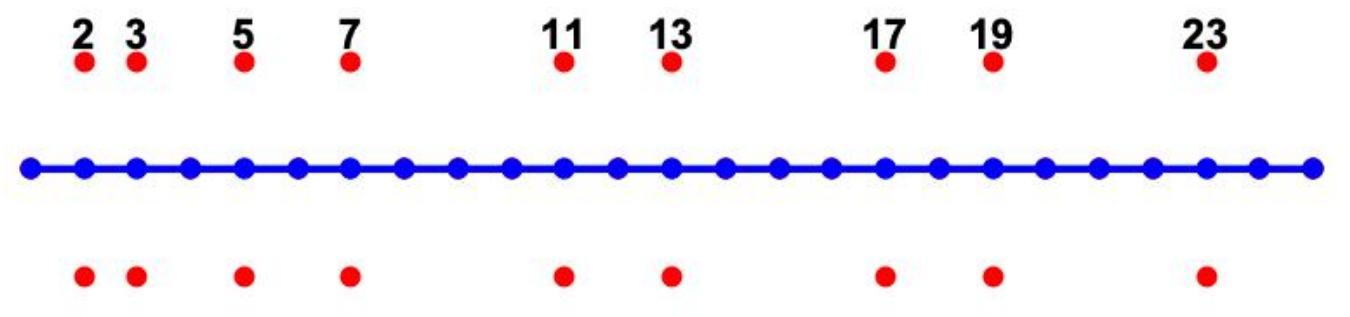

FiguRE 1. The natural numbers from 1 to 25 , with the first nine primes plotted (in red) at equal distances above and below.

2010 Mathematics Subject Classification. 11P32, 03B25.

Key words and phrases. Number Theory, Logic.

Received on 31-8-2020; revised 10-9-2020. 
In Fig. 2 we plot some lines joining primes in the upper row to primes in the lower row. Each such line intersects the central line at the mean value of the two primes. We see that there is a crossing line passing through every whole number: this is in agreement with the conjecture as expressed in (1).

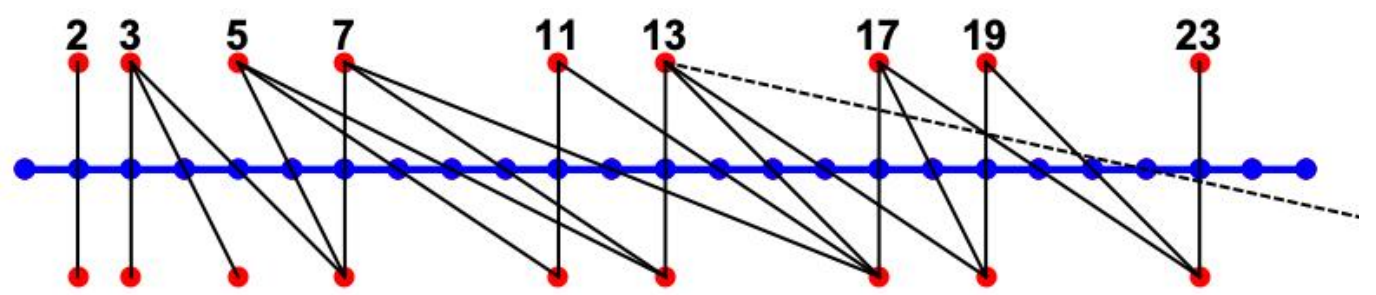

Figure 2 . The line from $p_{1}$ above to $p_{2}$ below crosses the number line at the mean value $\left(p_{1}+p_{2}\right) / 2$. There is an intersection of some line of this sort for every $n \geq 2$.

It is clear from the conjecture that every even number can be expressed in terms of primes in two distinct ways: as a sum of two primes and as the mean of two primes:

$$
\begin{aligned}
& \forall n \in \mathbb{N} \quad \exists p_{1}, p_{2} \in \mathbb{P}: \quad 2 n=p_{1}+p_{2} \\
& \forall n \in \mathbb{N} \quad \exists p_{3}, p_{4} \in \mathbb{P}: \quad 2 n=\left(p_{3}+p_{4}\right) / 2 .
\end{aligned}
$$

For the second of these forms, we have assumed that $4 n=p_{3}+p_{4}$.

\section{Uncle Petros and Goldbach's Conjecture}

The Goldbach Conjecture is the central theme of a novel by Apostolos Doxiadis, Uncle Petros and Goldbach's Conjecture, published in Greek in 1992 and in English in 2000. The hero is Petros Papachristos, a gifted, reclusive Greek mathematician who has spent most of his career trying to prove Goldbach's Conjecture. The narrator is his nephew, who tells the story of how, when he was a young teenager, his eccentric Uncle Petros set him the task of proving the conjecture.

The novel describes aspects of the recent history of mathematics, and gives some brilliant insights into the mental state and methods of a research mathematician. Although it is a work of fiction, Doxiadis gets the mathematical details right. He gives a great feeling for the passion that drives a research mathematician, and a good flavour of the nature of pure mathematics. He makes it clear that, while mathematical research is an enthralling and creative activity, it can become an obsession, obliterating all other interests, captivating the researcher and compelling him or her to work for years on a problem that may seem to others to be unimportant.

Several real-life mathematicians appear as characters in the book, including Constantin Carathéodory, G. H. Hardy, J. E. Littlewood, Srinivasa Ramanujan, Kurt Gödel and Alan Turing. The hero, Petros Papachristos, interacts with Hardy, Littlewood and Ramanujan, and he is profoundly affected by the work of Gödel and Turing. He realises the implication of advances in mathematical logic: Goldbach's Conjecture may be unprovable; the goal of his life's work may be unattainable.

To generate publicity for the book, the publishers offered a $\$ 1$ million prize to anyone who could prove Goldbach's Conjecture within two years of the date of publication. The prize was never claimed. 


\section{The Entscheidungsproblem}

Can every true mathematical statement be proved? The great German mathematician David Hilbert believed so and in 1928, together with Wilhelm Ackermann, he formulated the "Decision Problem", asking for an algorithm to establish a priori the validity or otherwise of any conjecture. He was destined to be disappointed.

In 1931 Kurt Gödel proved that mathematics is incomplete: whatever system of axioms we assume, there are statements that are true but that cannot be proved using only these axioms. Adding additional axioms may make such statements true but then new true-but-unprovable statements inevitably arise.

So, do we know of any statements that are unprovable using the usual axioms of mathematics? The Zermelo-Fraenkel (ZF) axioms of set theory form one of the standard starting-points. We now know that the axiom of choice $(\mathrm{C})$ is independent of $\mathrm{ZF}$, and that the continuum hypothesis is independent of $\mathrm{ZF}+\mathrm{C}$.

But what if we have a conjecture that we wish to prove, starting from the usual axioms of mathematics? Can we know in advance whether a mathematical proof is possible, or whether the conjecture is unprovable?

Hilbert's Decision Problem (Entscheidungsproblem) asks, in essence, if there is a way to determine - in the absence of a proof - whether any given mathematical statement or proposition is true or false. More specifically: Is there an algorithm that will take a logical statement in a formal language, and that will output its truth value. The algorithm does not need to indicate how it obtains the answer. Nor does it have to provide a proof. It just has to determine whether the statement is or is not valid.

In 1936, the American logician Alonzo Church showed that there can be no positive answer to the Entscheidungsproblem. Independently, and at about the same time, Alan Turing reached the same conclusion using a completely different method.

Church and Turing showed that it is impossible for an algorithm to decide in general whether a given statement in arithmetic is true or false. The implication of this is that, within a given system of axioms, there is no way to tell, ahead of time, whether a given conjecture can or cannot be proved. Hilbert's dream was shattered.

When Uncle Petros learned of these results, he too was devastated. He realised that a proof of Goldbach's Conjecture, on which he had laboured for decades, might not be possible. His life's work could have been in vain.

\section{UnProvable BUt True?}

We have no solid reason for suggesting that Goldbach's Conjecture cannot be proved on the basis of the usual axioms of mathematics. Although it is amusing to speculate, the only justification for such a claim is that the problem has been around for almost 280 years, and some of the most brilliant mathematicians have tried and failed to prove it. If a proof is found, it will likely involve some radically new idea or approach.

But let us suppose the conjecture is unprovable. Then it must be true!

Why? Because, if it is false, there exists an even number $B$ that is not the sum of two primes. Since partitioning into primes has been confirmed for numbers up to more than a million million million, $B$ must be enormous. However, it is finite, and a finite search must confirm that there are no two primes that add to $B$.

This would make the conjecture "provably false". In other words, falsehood of the conjecture is incompatible with unprovability. This contradiction forces us to an ineluctable conclusion: if Goldbach's Conjecture is unprovable, it must be true! 


\section{REFERENCES}

[1] Doxiadis, Apostolos, 2000: Uncle Petros and Goldbach's Conjecture. Faber \& Faber, London. ISBN: 978-0-5712-0511-0.

[2] Wikipedia article Goldbach's Conjecture. https://en.wikipedia.org/wiki/Goldbach\%27s_conjecture (accessed 01-09-2020)

[3] Wikipedia article The Entscheidungsproblem. https://en.wikipedia.org/wiki/Entscheidungsproblem (accessed 01-09-2020)

Peter Lynch is emeritus professor at UCD. His interests include all areas of mathematics and its history. He writes an occasional mathematics column in The Irish Times and has published a book of articles entitled Thats Maths. His blog is at http://thatsmaths.com.

School of Mathematics \& Statistics, University College Dublin

E-mail address: Peter.Lynch@ucd.ie 